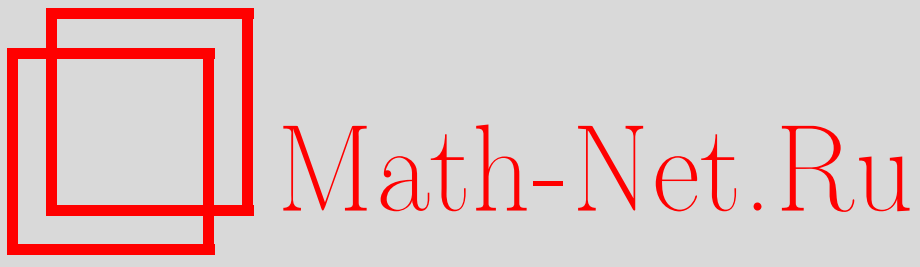

А. М. Вершик, Г. А. Фрейман, Ю. В. Якубович, Локальная предельная теорема для случайных разбиений натуральных чисел, Теория вероятн. и ее примен., 1999, том 44, выпуск $3,506-525$

DOI: https://doi.org/10.4213/tvp801

Использование Общероссийского математического портала Math-Net.Ru подразумевает, что вы прочитали и согласны с пользовательским соглашением http://www . mathnet.ru/rus/agreement

Параметры загрузки:

IP : 54.174 .149 .18

26 апреля 2023 г., 05:44:20

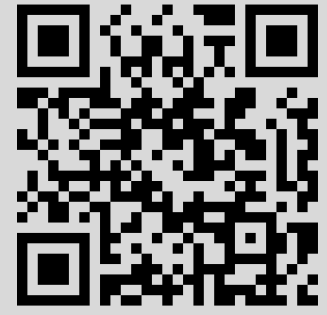


(C) 1999 г.

\author{
ВЕРШИК А. М.*, \\ ФРЕЙМАН Г. А. ${ }^{* *}$, ЯКУБОВИЧ Ю. В.* \\ ЛОКАЛЬНАЯ ПРЕДЕЛЬНАЯ ТЕОРЕМА \\ ДЛЯ СЛУЧАЙНЫХ РАЗБИЕНИЙ \\ НАТУРАЛЬНЫХ ЧИСЕЛ ${ }^{1}$ )
}

\begin{abstract}
Рассматривается множество разбиений натурального числа $n$ на различные слагаемые, снабженное равномерным распределением. Помимо задачи о предельной форме типичного разбиения при $n \rightarrow \infty$, изучавшейся в [20], представляет интерес поведение флюктуаций разбиений около предельной формы. Переход на геометрический язык позволяет свести задачу к изучению предельного поведения случайных ступенчатых функций (диаграмм Юнга). В работе доказаны утверждения типа локальной предельной теоремы, из которых следует, что совместные распределения флюктуаций в нескольких точках локально асимптотически нормальны. Метод работы существенно использует понятие большого канонического ансамбля разбиений.
\end{abstract}

Клточевые слова и фразы: разбиение, диаграмма Юнга, большой ансамбль разбиений, предельная форма, локальная предельная теорема.

Создателем теории разбиений натуральных чисел был первый российский профессиональный математик Леонард Эйлер (1707-1783), прибывший в 1727 году в С.-Петербург по рекомендации братьев Бернулли в качестве адъюнкта только что образованной (1724 г.) Петром Первым Российской Академии наук и ставший впоследствии одним из самых выдаюшихся математиков своего века. Теорию разбиений и, более общо, аддитивные задачи теории чисел он называл «Partitio Numerorum» и с энтузиазмом занимался ею на протяжении многих лет.

${ }^{*}$ С.-Петербургское отделение Математического института им. В.А. Стеклова РАН, ул. Фонтанка, 27, 191011 С.-Петербург, Россия.

**The Raymond and Beverly Sackler Faculty of Exact Sciences, Tel Aviv University, Tel Aviv, Israel.

1) Работа выполнена при частичной поддержке Российским фондом фундаментальных исследований, грант 96-01-00676, первого и третьего авторов. 
Он дал прежде всего классическую формулу для производящей функции числа разбиений:

$$
\prod_{k=1}\left(1-x^{k}\right)^{-1}=\sum_{n=0} p(n) x^{n}
$$

нашел первые нетривиальные тождества, связанные с разбиениями, тождество Эйлера - предшественник тождеств Гаусса-Якоби, тождества с разбиениями на неравные слагаемые (именно этот предмет изучается и в настоящей статье) и т.д. В недавно вышедшей книге «Неопубликованные материалы Л. Эйлера по теории чисел» [7] есть глава, посвященная неизвестным работам Эйлера по теории разбиений.

Дальнейпий прогресс в этой теории был обязан, с одной стороны, мошным аналитическим методам (Харди, Рамануджан, Радемахер), а также глубоким связям с теорией модулярных форм, а с другой - применению разбиений и связанных с ними диаграмм Юнга в комбинаторике, теории представлений, геометрии и т.д. К настоящему времени эти связи и применения сделали теорию разбиений одной из самых популярных теорий.

Неудивительно, что в надлежащий момент (а именно, около двух десятилетий назад) появилась и вероятностная теория разбиений. При этом, она рождена была не из «обобщательских» соображений, а по причине естественного появления статистик на разбиениях, возникавших как в самой теории чисел, так и в теории представлений (случайные диаграммы Юнга), геометрии, и, что еще более удивительно, - из параллелей со статистической физикой.

В этой работе рассматривается наиболее естественная статистика на разбиениях с различными слагаемыми - равномерная, т.е. статистика Ферми. Исследованием асимптотик распределений тех или иных функционалов относительно этой статистики занималась школа Эрдеша в 40-60-х гг. В работах [20]-[22] задача ставилась по-новому, как задача об одновременном описании асимптотики всех однотипных функционалов или как задача о предельной форме разбиения и его диаграммы Юнга. Предельная форма для этого случая была найдена ранее, а в настоящей работе мы изучаем поведение флюктуаций около предельной формы.

1. Постановка задачи и основные результаты. В этой работе исследуется поведение флюктуаций числа слагаемых, бо́льших данного, в случайном разбиении натурального числа на различные слагаемые относительно равномерного распределения на множестве всех таких разбиений.

Пусть $n$ - натуральное число и $\mathscr{P}^{d}(n)=\mathscr{P}(n)$ - множество всех неупорядочениых разбиений числа $n$ на различные слагаемые. Такое 
разбиение $\lambda$ однозначно определяется числами заполнения $\rho_{k}(\lambda)$ по правилу $\rho_{k}(\lambda)=1$, если число $k$ есть одно из слагаемых в разбиении $\lambda$, и $\rho_{k}(\lambda)=0$ в противном случае; при этом $n=\sum_{k=1}^{n} k \rho_{k}$.

Снабдим $\mathscr{P}(n)$ равномерной мерой $\mu^{n}$; числа заполнения можно рассматривать как случайные величины на $\left(\mathscr{P}(n), \mu^{n}\right)$; при этом $\mu^{n}(\lambda)=$ $(q(n))^{-1}, q(n)=\# \mathscr{P}(n) ; F(x)=\sum_{n=0}^{\infty} q(n) x^{n}=\prod_{k=1}^{\infty}\left(1+x^{k}\right)$ - известная производящая функция для чисел $\{q(n)\}$, см. [1].

Общая задача состоит в изучении асимптотического поведения чисел заполнения типичного разбиения при росте разбиваемого числа. Наиболее плодотворная постановка вопроса, предложенная в [20]-[22], такова. Сопоставим каждому разбиению $\lambda \in \mathscr{P}(\cdot)$ функцию

$$
g_{\lambda}(t)=\sum_{k \geqslant t} \rho_{k}(\lambda) .
$$

Подграфик этой функции есть диаграмма Юнга, отвечающая этому разбиению. Таким образом, $g_{\lambda}(\cdot)$ есть кусочно-постоянная случайная (по $\lambda \in \mathscr{P}(n))$ функщия.

Если отнормировать эти функции следующим образом:

$$
\widetilde{g}_{\lambda}(t)=\frac{\pi}{2 \sqrt{3 n}} g_{\lambda}\left(\frac{2 \sqrt{3 n}}{\pi} t\right)
$$

(множитель $\pi /(2 \sqrt{3})$ выбран для удобства), то, оказывается, имеет место следуюший закон больших чисел:

$$
\lim _{n \rightarrow \infty} \mu^{n}\left\{\lambda \in \mathscr{P}(n):\left|\tilde{g}_{\lambda}(t)-g(t)\right|<\varepsilon\right\}=1,
$$

где $g(t)=\ln \left(1+e^{-t}\right)\left(\right.$ или $\left.e^{g(t)}-e^{-t}=1\right)$, найденный в [20].

График функции $g(\cdot)$ есть предельная форма («limit shape»), при $n \rightarrow \infty$, случайных диаграмм Юнга, отвечағщих разбиениям.

В работах [6] и [4] доказаны теоремы о больших уклонениях в той же задаче.

Целью данной работы является доказательство локальных теорем в той же постановке. Более точно, рассмотрим $\widetilde{g}_{\lambda}(t)-$ нормированное число слагаемых разбиения $\lambda$, не меньших, чем $t \pi /(2 \sqrt{3 n})$. Из цитированного выше результата следует (мы передоказываем это ниже), что $\lim _{n \rightarrow \infty} \mathbf{E} \widetilde{g}_{\lambda}(t)=\ln \left(1+e^{-t}\right)$. Ставится вопрос о нахождении дисперсии $\mathbf{D} \widetilde{g}_{\lambda}(t)$ и о поведении $\left(\sqrt{\mathbf{D} \widetilde{g}_{\lambda}(t)}\right)^{-1}\left(\widetilde{g}_{\lambda}(t)-\mathbf{E} \widetilde{g}_{\lambda}(t)\right)$. Мы доказываем нормальность предельного распределения:

Теорема 1. Пусть $t \geqslant 0, a>b-$ произвольные вещественные числа. Тогда при $n \rightarrow \infty$

$$
\mu^{n}\left\{\lambda \in \mathscr{P}(n): \frac{\tilde{g}_{\lambda}(t)-g(t)}{\sqrt{d(t) \sigma}} \in(a, b)\right\}=\frac{1}{\sqrt{2 \pi}} \int_{a}^{b} e^{-\frac{x^{2}}{2}} d x(1+O(\sqrt{\sigma})),
$$


zде $\sigma=\pi /(2 \sqrt{3 n}), a$

$$
d(t)=\frac{1}{1+e^{t}}-\frac{6}{\pi^{2}}\left(\frac{t}{1+e^{t}}+\ln \left(1+e^{-t}\right)\right)^{2} .
$$

В теореме 1 доказывается гауссовость флюктуаций соответствующим образом нормированной диаграммы Юнга в каждой точке, т.е. гауссовость одномерных распределений. Она следует из более сильной локальной предельной теоремы для флюктуаций диаграммы Юнга в нескольких точках:

Теорема 2. Пусть $d>0$ - натуральное число. Рассмотрим $d$ натуральных чисел $m_{1}, m_{2}, \ldots, m_{d}$, зависящих от $n$ так, что $m_{i} / \sqrt{n} \in$ $[a, b], 0<a<b<\infty ;$ обозначим $M=\left(m_{1}, m_{2}, \ldots, m_{d}\right)$. Пусть $0 \leqslant t_{1}<t_{2}<\cdots<t_{d}-$ вещественные числа; обозначим $\widehat{g}_{\lambda}(\mathbf{t})=$ $\left(g_{\lambda}\left(t_{1} / \sigma\right), \ldots, g_{\lambda}\left(t_{d} / \sigma\right)\right) u g(\mathrm{t})=\left(g\left(t_{1}\right), \ldots, g\left(t_{d}\right)\right)$, аде $\sigma=\pi /(2 \sqrt{3 n})$. Toгда при любых $а$ u $b$

$$
\begin{aligned}
\mu^{n}\{\lambda & \left.\in \mathscr{P}(n): \widehat{g}_{\lambda}(\mathbf{t})=M\right\}=\frac{1}{(2 \pi)^{d / 2} \sqrt{|D|}} \\
& \times \exp \left(-\frac{1}{2}\left\langle\frac{\sigma M-g(\mathbf{t})}{\sqrt{\sigma}}, D^{-1} \frac{\sigma M-g(\mathbf{t})}{\sqrt{\sigma}}\right\rangle\right)(1+O(\sigma)),
\end{aligned}
$$

где матрища $D=\left(e_{i j}\right)_{i, j=1}^{d}$ определяется следующим образом:

$$
e_{i j}=\frac{1}{1+e^{t_{k}}}-\frac{6}{\pi^{2}} h\left(t_{i}\right) \dot{h}\left(t_{j}\right)
$$

где $k=\max \{i, j\} \quad u h(t)=t /\left(1+e^{t}\right)+\ln \left(1+e^{-t}\right)$.

Поясним обозначения, примененные в формулировке теоремы. Вектор $g(\mathbf{t})$ есть вектор математических ожиданий значений верхней границы нормированной диаграммы Юнга в точках $t_{1}, \ldots, t_{d}$, а $\widehat{g}_{\lambda}(\mathbf{t})$ есть вектор значений в тех же точках верхней границы диаграммы Юнга, отвечаюшей разбиению $\lambda$, сжатой в $1 / \sigma$ раз вдоль оси абсцисс.

Следствие 1. При $s<t$ ковариачия между случайными величинами $\sqrt{\sigma} g_{\lambda}(s / \sigma) u \sqrt{\sigma} g_{\lambda}(t / \sigma)$ (по отношению $\kappa$ мере $\mu^{n}$ ) при $n \rightarrow \infty$ ecmb

$$
\begin{aligned}
\operatorname{Cov} & (n) \\
= & \left(\sqrt{\sigma} g_{\lambda}\left(\frac{s}{\sigma}\right), \sqrt{\sigma} g_{\lambda}\left(\frac{t}{\sigma}\right)\right) \\
& \quad \times\left(1+e^{t}-\frac{6}{\pi^{2}}\left(\frac{s}{1+e^{s}}+\ln \left(1+e^{-s}\right)\right)\left(\frac{t}{1+e^{t}}+\ln \left(1+e^{-t}\right)\right)\right) \\
& \times(\sqrt{\sigma}),
\end{aligned}
$$

2де $\sigma=\pi /(2 \sqrt{3 n})$.

Особо интересна асимптотика этой ковариации при близких $s$ и $t$. Простое вычисление показывает, что верно следующее утверждение. 
Следствие 2. Дисперсия разности. $\sqrt{\sigma} g_{\lambda}((t+\varepsilon) / \sigma)-\sqrt{\sigma} g_{\lambda}(t / \sigma)$ no отношению к мере $\mu^{n}$ при $n \rightarrow \infty$ может быть записана следующим образом:

$$
\lim _{n \rightarrow \infty} \mathbf{D}^{(n)}\left(\sqrt{\sigma} g_{\lambda}(t+\varepsilon)-\sqrt{\sigma} g_{\lambda}(t)\right)=\frac{e^{t}}{\left(1+e^{t}\right)^{2}} \varepsilon+O\left(\varepsilon^{2}\right) .
$$

Теорема 2 еше не дает возможности установить полный принцип инвариантности. Однако использование неполных преобразований Фурье цает возможность доказать многомерную предельную теорему. Она будет доказана в другой работе.

ІІрежде чем перейти к изложению методики и техники доказательства приведенных выше утверждений, напомним, что помимо непосредственного интереса описанные результаты имеют статфизическую интерпретацию: эти теоремы фактически описывают поведение распределения энергии идеального квантового Ферми-газа по импульсам, см. [21].

2. Большой и малый ансамбли разбиений. Доказательство теорем 1 и 2 основано на исследовании соотношения между мерами $\mu^{n}$ и гиббсовскими мерами $\mu_{x}$, к определению которых мы переходим.

ІІусть $\mathscr{P}^{d}=\mathscr{P}=\cup_{n \geqslant 0} \mathscr{P}(n)-$ множество разбиений всех $(\mathscr{P}(0)=$ $\{\varnothing\})$ натуральных чисел. Будем называть множество $\mathscr{P}$ большим ансамблем разбиений, а $\mathscr{P}(n)$ - мальм ансамблем разбиений числа $n$. Числа заполнения $\rho_{k}$ можно рассматривать как функции на большом ансамбле $\mathscr{P}$, и каждую меру $\mu^{n}$ можно также рассматривать как меру на $\mathscr{P}$.

Рассмотрим семейство выпуклых комбинаций мер $\mu^{n}$

$$
\mu_{x}=\frac{1}{F(x)} \sum_{n=0}^{\infty} q(n) x^{n} \mu^{n},
$$

где $x \in(0,1), q(n)=\# \mathscr{P}(n)$ и $F(x)=\prod_{k=1}^{\infty}\left(1+x^{k}\right)$. Меры $\mu_{x}$ обладают свойствами:

(1) $\left.\mu_{x}\right|_{\mathscr{P}(n)}=\left(q(n) x^{n} / F(x)\right) \mu^{n}$, т.е. ограничение мер $\mu_{x}$ на $\mathscr{P}(n)$ при всех $x \in(0,1)$ с точностью до множителя совпадает с мерами $\mu^{n}$;

(2) числа заполнения $\left\{\rho_{k}\right\}_{k=1}^{\infty}$ независимы относительно мер $\mu_{x}$ при всех $x \in(0,1)$.

Свойство (1) следует мгновенно из определения. Докажем свойство (2). Действительно, рассмотрим финитную последовательность $j_{1}, j_{2}, \ldots ; j_{k} \in\{0,1\}$ и обозначим $n=\sum_{k} k j_{k}$. Тогда

$$
\begin{aligned}
& \mu_{x}\left\{\rho_{1}=j_{1}, \rho_{2}=j_{2}, \ldots\right\}=\frac{1}{F(x)} q(n) x^{n} \mu^{n}\left\{\rho_{1}=j_{1}, \rho_{2}=j_{2}, \ldots\right\} \\
& =\frac{1}{F(x)} q(n) x^{\sum k j_{k}} \frac{1}{q(n)}=\prod_{k=1}^{\infty} \frac{x^{k j_{k}}}{1+x^{k}},
\end{aligned}
$$

что и означает независимость чисел заполнения. 
Семейство мер $\left\{\mu^{n}\right\}$, для которого сушествует семейство $\mu_{x}$, обладающее свойствами (1), (2), называют мультипликативным семейством [20]. Меры $\mu_{x}$ естественно назвать гиббсовскими. Из доказательства свойства (2) следует, что в нашем случае

$$
\mu_{x}\left\{\lambda \in \mathscr{P}: \rho_{k}(\lambda)=0\right\}=\frac{1}{1+x^{k}} \quad \text { и } \quad \mu_{x}\left\{\lambda \in \mathscr{P}: \rho_{k}(\lambda)=1\right\}=\frac{x^{k}}{1+x^{k}} .
$$

Заметим, что независимость не имеет места на $\left(\mathscr{P}(n), \mu^{n}\right)$. Независимость чисел заполнения относительно $\mu_{x}$ позволяет использовать стандартные методы теории вероятностей для нахождения асимптотики на большом ансамбле, а затем перенести полученные результаты на малый ансамбль разбиений. Тем самым, наш метод исследования асимптотики мер $\mu^{n}$ состоит в замене их мерами $\mu_{x}$ и в оправдании возможности такой замены. Этот метод имеет давнюю историю и разнообразные формулировки. В статистической механике он возник как способ обоснования эквивалентности болышого и малого канонических ансамблей. Аналитическая его сторона обрела формулировку метода перевала (метода Дарвина-Фаулера) или ВКБ (см. [12] и [16]). С другой стороны, в анализе и теории чисел аналогичные комплексные методы систематически использовались для нахождения асимптотики коэффициентов ряда Тейлора. Независимо от этого в теории вероятностей к таким же проблемам приводит метод характеристических функций для доказательства локальных предельных теорем (теорема Г. Крамера). А.Я. Хинчин [13], [14] был, по-видимому, первым, кто обратил внимание на это сходство и применил простейшие локальные теоре мы для обоснования результатов квантовой статистической физики. В более поздних работах различные варианты теоретико-вероятностного подхода были использованы в теоретико-числовых (Г.А. Фрейман [8], [9], см. также указанную там литературу), комбинаторных (R. Arratia и S. Tavaré [2], В. Fristedt [11], Ю.В. Якубович [23]), геометрических (А.М. Вершик [22], Я.Г. Синай [18]) и других задачах. Близкие вероятностные задачи комбинаторного происхождения рассматривались в работах В.Ф. Колчина и В.ІІІ. Чистякова [15], В.Н. Сачкова [19] и др.

В этой работе мы доказываем утверждения о флюктуациях числа слагаемых, больших данного, аналогичные классическим локальным предельным теоремам, но несколько отличающиеся от последних и по постановке, и по методу доказательств. Мы используем технику большого канонического ансамбля и метод, аналогичный [9], и применяем их к задаче о флюктуации около предельной формы («limit shape») разбиения - см. [20]. Насколько нам известно, подобные постановки ранее не рассматривались. С другой стороны следует отметить, что хотя параллелизм между статфизическими, комплексными и вероятностными 
методами (см. выше) очевиден, взаимоотношения между ними исследованы до. сих пор недостаточно.

Мы будем использовать обозначения $\mathrm{E}_{x} \xi$ и $\mathrm{D}_{x} \xi$ для математического ожидания и матрицы ковариаций (дисперсии в одномерном случае) слуучайного вектора $\xi$, взятых по отношению к гиббсовской мере $\mu_{x}$.

3. Доказательство теорем 1 и 2. Теорема 1 следует из утверждения теоремы 2 при $d=1$ : достаточно просуммировать $O(\sqrt{\sigma})$ асимптотических выражений для вероятности $\widehat{g}_{\lambda}(t)=m$ при $m \in(a / \sqrt{\sigma}, b / \sqrt{\sigma})$. Сформулируем утверждение теоремы 2 в нужной нам форме. Мы используем обозначения, введенные в ее формулировке. Пусть, как и ранее, $\sigma=\pi /(2 \sqrt{3 n})$. Обозначим разбиваемое число (случайное по отношению к мере $\left.\mu_{x}\right)$

$$
\mathbf{n}(\lambda)=\int_{0}^{\infty} g_{\lambda}(t) d t=\sum_{k=0}^{\infty} k \rho_{k}(\lambda)
$$

Интересующие нас меры $\mu^{n}$ и $\mu_{x}$ при всех $x \in(0,1)$ связаны следующим соотношением, являюшимся формулой для условной вероятности:

$$
\mu^{n}\left\{\lambda \in \mathscr{P}(n): \widehat{g}_{\lambda}(\mathbf{t})=M\right\}=\frac{\mu_{x}\left\{\lambda \in \mathscr{P}: \mathbf{n}(\lambda)=n, \widehat{g}_{\lambda}(\mathbf{t})=M\right\}}{\mu_{x}\{\lambda \in \mathscr{P}: \mathbf{n}(\lambda)=n\}} .
$$

Если бы правая часть этого равенства не содержала $\sigma$, зависящего от $n$, то эти уравнения позволили бы нам сразу перейти к изучению мер $\mu_{x}$ вместо мер $\mu^{n}$. Однако $\sigma$ неявно содержится в правой части (1) (в определении $\left.\widehat{g}_{\lambda}(\mathrm{t})\right)$, но мы можем заменить $n$ на математическое ожидание разбиваемого числа $\mathbf{n}(\lambda)$ и выбрать зависимость $x$ от $n$ таким образом, чтобы это математическое ожидание в точности равнялось $n$. В таком случае правая часть (1) формально не зависит от $n$, и мы можем исследовать соответствующие распределения. Мы собираемся применить локальную предельную теорему к правым частям (1), проверив, что она выполнена для соответствуюших случайных величин. Как уже было отмечено выше, чтобы получить правильный результат, мы должны выбирать $x=x(n)$ таким, что

$$
\mathbf{E}_{x} \mathbf{n}=\mathbf{E}_{x} \sum_{k=1}^{\infty} k \rho_{k}=\sum_{k=1}^{\infty} \frac{k x^{k}}{1+x^{k}}=n .
$$

Таким образом, для доказательства теоремы 2 мы должны найти асимптотику правой части соотношения (1). При этом мы применяем локальную предельную теорему в большом ансамбле разбиений, доказываемую в следуюшем пункте. Пока приведем лишь ее формулировку.

Tеорема 3. Пусть $d \in \mathbf{Z}_{+}=\{0,1,2, \ldots\}, n \in \mathbf{N} u M=M(x)=$ $\left(m_{1}, \ldots, m_{d}\right)$ есть $d$-мерный вектор с неотричательными чельми компонентами, зависящий от $x \operatorname{max,~что~} m_{i} /|\ln x| \in[a, b], 0<a<$ 
$b<\infty$. Обозначим $N=(n, M) u \bar{N}=N-\mathbf{E}_{x}\left(\mathbf{n}(\lambda), \widehat{g}_{\lambda}(\mathbf{t})\right)$. Тогда $n p u$ любых $a$ u $b$

$$
\begin{aligned}
& \mid \mu_{x}\left\{\lambda \in \mathscr{P}: \mathbf{n}(\lambda)=n, \widehat{g}_{\lambda}(\mathbf{t})=M\right\} \\
& \quad-\frac{1}{(2 \pi)^{(d+1) / 2} \sqrt{|D|}} \exp \left(-\frac{\left\langle\bar{N}, D^{-1} \bar{N}\right\rangle}{2}\right) \mid=O(|\ln x|) .
\end{aligned}
$$

при $x \rightarrow 1$, где $D=\mathbf{D}_{x}\left(\mathbf{n}(\lambda), \widehat{g}_{\lambda}(\mathbf{t})\right)-$ матрича ковариачий случайного вектора $\left(\mathbf{n}(\lambda), \widehat{g}_{\lambda}(\mathbf{t})\right)$.

Ниже мы считаем, что рассматриваемая на $\mathscr{P}$ мера есть $\mu_{x}$, если не оговорено противное. Следуюшая лемма объясняет асимптотику совместного распределения величин $\left(\mathbf{n}(\lambda), \widehat{g}_{\lambda}(\mathbf{t})\right)$.

Лемма 1. Пусть $x \in(0,1)$. Тогда при $x \rightarrow 1-0$ верньи следующие асимптотические формулы для математических ожиданий по отношению $\kappa$ мере $\mu_{x}$ :

$$
\mathbf{E}_{x} \mathbf{n}=\frac{\pi^{2}}{12 \sigma^{2}}(1+O(\sigma)) ; \quad \mathbf{E}_{x} g_{\lambda}(t / \sigma)=\frac{1}{\sigma} g(t)(1+O(\sigma)) ;
$$

и для матричы ковариачий:

$$
\begin{aligned}
D & =\mathbf{D}_{x}\left(\mathbf{n}(\lambda), \widehat{g}_{\lambda}(\mathbf{t})\right) \\
& =\left(\begin{array}{ccccc}
\frac{1}{\sigma^{3}} \frac{\pi^{2}}{6} & \frac{1}{\sigma^{2}} h\left(t_{1}\right) & \frac{1}{\sigma^{2}} h\left(t_{2}\right) & \cdots & \frac{1}{\sigma^{2}} h\left(t_{d}\right) \\
\frac{1}{\sigma^{2}} h\left(t_{1}\right) & \frac{1}{\sigma} \frac{1}{1+e^{t_{1}}} & \frac{1}{\sigma} \frac{1}{1+e^{t_{2}}} & \cdots & \frac{1}{\sigma} \frac{1}{1+e^{t_{d}}} \\
\frac{1}{\sigma^{2}} h\left(t_{2}\right) & \frac{1}{\sigma} \frac{1}{1+e^{t_{2}}} & \frac{1}{\sigma} \frac{1}{1+e^{t_{2}}} & \cdots & \frac{1}{\sigma} \frac{1}{1+e^{t_{d}}} \\
\vdots & \vdots & \vdots & \ddots & \vdots \\
\frac{1}{\sigma^{2}} h\left(t_{d}\right) & \frac{1}{\sigma} \frac{1}{1+e^{t_{d}}} & \frac{1}{\sigma} \frac{1}{1+e^{t_{d}}} & \cdots & \frac{1}{\sigma} \frac{1}{1+e^{t_{d}}}
\end{array}\right)(1+O(\sigma)),
\end{aligned}
$$

гдe $\sigma=-\ln x u h(t)=t /\left(1+e^{t}\right)+\ln \left(1+e^{-t}\right)$.

Д о к а з а т е л ь с т в о. Мы детально проследим только за доказательством первого равенства, поскольку доказательства остальных отличаются от первого только тем, какие интегралы необходимо взять. Математическое ожидание $\mathbf{n}$ может быть записано в следующем виде:

$$
\mathbf{E}_{x} \mathbf{n}=\mathbf{E}_{x} \sum_{k=1}^{\infty} k \rho_{k}=\sum_{k=1}^{\infty} k \mathbf{E}_{x} \rho_{k}=\sum_{k=1}^{\infty} \frac{k x^{k}}{1+x^{k}}
$$

Сделаем замену переменной $\sigma=-\ln x ;$ когда $x \rightarrow 1$ слева, то $\sigma \rightarrow 0$ справа. (Отметим, что $\sigma$ здесь формально не связана с $n$, как это было выше и к чему мы еще вернемся.) С использованием этой новой переменной 
выражение для $\mathbf{E}_{x} \mathbf{n}$ может быть переписано как

$$
\mathbf{E}_{x} \mathbf{n}=\frac{1}{\sigma} \sum_{k=1}^{\infty} \frac{k \sigma e^{-k \sigma}}{1+e^{-k \sigma}}=\underbrace{\frac{1}{\sigma} \sum_{k=1}^{[M / \sigma]} \frac{k \sigma e^{-k \sigma}}{1+e^{-k \sigma}}}_{S_{1}}+\underbrace{\sum_{k=[M / \sigma]+1}^{\infty} \frac{k x^{k}}{1+x^{k}}}_{S_{2}}
$$

шри произвольной константе $M$. Нас интересует асимптотика этой суммы при $\sigma \rightarrow 0+$. Рассмотрим функцию $f(z)=z e^{-z} /\left(1+e^{-z}\right)$. Тогда $\sigma^{2} S_{1}$ есть сумма Римана для интеграла

$$
\int_{0}^{M} f(z) d z=-M \ln \left(1+e^{-M}\right)+\int_{e^{-M}}^{1} \frac{\ln (1+z)}{z} d z .
$$

Поскольку $M$ - произвольно большое число, то этот интеграл может быть сделан сколь угодно близким к своему пределу при $M \rightarrow \infty$, а именно дилогарифму -1 , равному $-\mathrm{Li}_{2}(-1)=\pi^{2} / 12$. Сумма $S_{2}$ может быть, в свою очередь оценена (при больших $M$ и $x \in(0,1)$ ) слепуюшим образом:

$$
S_{2} \leqslant \sum_{k=[M / \sigma]}^{\infty} k x^{k}=\frac{x^{[M / \sigma]}([M / \sigma](x-1)+x)}{(x-1)^{2}} \leqslant C \frac{M e^{-M}}{\sigma^{2}}
$$

Таким образом, в силу произвольности $M, \mathbf{E}_{x} \mathbf{n}=\pi^{2}\left(12 \sigma^{2}\right)^{-1}(1+O(\sigma))$.

Все остальные утверждения леммы проверяются совершенно аналогично, и детальная проверка оставляется читателю.

Теперь мы можем выяснить, как нужно выбирать зависимость $x$ от $n$. Прежде всего заметим, что уравнение (2) всегда имеет единственное решение на $x \in(0,1)$, поскольку $\mathbf{E}_{x} \mathbf{n}$ есть сумма строго возрастаюших функций $k \mathbf{E}_{x} \rho_{k}=k x^{k} /\left(1+x^{k}\right)$ и, к тому же, неограниченно возрастает при $x \rightarrow 1$. Для получения асимптотики необязательно иметь точное решение, и, используя первое утверждение леммы 1, мы можем выбрать

$$
\sigma=-\ln x=\frac{\pi}{2 \sqrt{3 n}}
$$

При таком выборе $x=x(n)$ уравнение (2) выполнено асимптотически; асимптотическая формула для точного решения есть $\ln x=-\pi /(2 \sqrt{3 n})+$ $O(1 / n)$. До конща этого пункта мы будем предполагать, что соотношение (4) выполнено. (Заметим, что таким образом мы фиксируем зависимость $x$ от $n$, а $\sigma$ является параметром, введенным лишь для сокращения обозначений, и может быть выбрано зависяпим как от $x$, так и от $n$ ). При $n \rightarrow \infty$ последовательность $x=x_{n}$ стремится к 1 слева. Поэтому общие результаты о поведении распределений случайных величин при произвольном стремлении $x$ к 1 слева, в частности, теорема 3 , верны и для последовательности $x=x_{n}$. 
Рассматривая $\sigma$ в (1) как функцию от $x$, мы можем применять ло кальную предельную теорему 3 к числителю и знаменателю правой части соотношения (1). Для упрощения получаемых формул нам потребуется следующее элементарное алгебраическое утверждение.

Лемма 2. Пусть $a, \tilde{a} \in \mathbf{R}, B, \widetilde{B} \in \mathbf{R}^{d}$ (мы считаем $B$ u $\widetilde{B}$ векторами-столбиами) и $C, \widetilde{C}$ - симметричные матрицы $d \times d$, где $d$ - некоторое натуральное число, возможно 1. Пусть они связаны следующим соотношением:

$$
\left(\begin{array}{cc}
a & B^{T} \\
B & C
\end{array}\right)^{-1}=\left(\begin{array}{cc}
\widetilde{a} & \widetilde{B}^{T} \\
\widetilde{B} & \widetilde{C}
\end{array}\right)
$$

Тогда: 1) если $C$ обратима, то $\widetilde{C}$ также обратима, и обратная к ней матрица может быть вычислена по форлуле $\widetilde{C}^{-1}=C-a^{-1} B B^{T}$;

2) определитель

$$
\operatorname{det}\left(\begin{array}{cc}
a & B^{T} \\
B & C
\end{array}\right)=a \operatorname{det}\left(C-\frac{1}{a} B B^{T}\right) .
$$

Д о к а з а т е л с т в о. Докажем сначала первое утверждение. Предположим, что $C$ обратима. Рассмотрим $x, \widetilde{x} \in \mathbf{R}, y, \widetilde{y} \in \mathbf{R}^{d}$ такие, ч'To

$$
\left(\begin{array}{cc}
a & B^{T} \\
B & C
\end{array}\right)\left(\begin{array}{l}
x \\
y
\end{array}\right)=\left(\begin{array}{l}
\tilde{x} \\
\tilde{y}
\end{array}\right) \text {. }
$$

Тогда

$$
\left(\begin{array}{cc}
\widetilde{a} & \widetilde{B}^{T} \\
\widetilde{B} & \widetilde{C}
\end{array}\right)\left(\begin{array}{l}
\widetilde{x} \\
\widetilde{y}
\end{array}\right)=\left(\begin{array}{l}
x \\
y
\end{array}\right) .
$$

Из системы (6) получаем $\widetilde{x}=a x+B^{T}\left(C^{-1} \widetilde{y}-B x\right)$, или $\widetilde{x}-B^{T} C^{-1} \widetilde{y}=$ $\left(a-B^{T} C^{-1} B\right) x$. Обозначим $r=a-B^{T} C^{-1} B$ и заметим, что $r$ - число. Если $r=0$, то нетрудно проверить, что $\operatorname{det}\left(\begin{array}{cc}a & B^{T} \\ B & C\end{array}\right)=0$, что противоречит условию (5). Поэтому $r \neq 0$, и $x=r^{-1} \widetilde{x}-r^{-1} B^{T} C^{-1} \widetilde{y}$. Выражая $y$ из системы (6) и подставляя найденное выражение для $x$, получаем $y=C^{-1}(\widetilde{y}-B x)=C^{-1} \widetilde{y}-r^{-1} C^{-1} B \widetilde{x}+r^{-1} C^{-1} B B^{T} C^{-1} \widetilde{y}$. Поскольку $\left(\begin{array}{l}x \\ y\end{array}\right)$ - прокзвольный вектор в $\mathbf{R}^{d+1}$, это означает, что $\widetilde{C}=C^{-1}\left(I+r^{-1} B B^{T} C^{-1}\right)$, где $I-$ единичная матрица $d \times d$. Отсюда получаем $\widetilde{C}^{-1}=\left(I+r^{-1} B B^{T} C^{-1}\right)^{-1} C$. Теперь нам понадобится тождество $\left(I+f g^{T}\right)^{-1}=I-f g^{T} /\left(1+g^{T} f\right)$, справедливое для любых векторов $f$ и $g$, которое легко проверить, перемножив левую и правую части. Используя его при $f=r^{-1} B$ и $g^{T}=B^{T} C^{-1}$, получаем

$$
\widetilde{C}^{-1}=C-\frac{r^{-1} B B^{T} C^{-1} C}{1+r^{-1} B^{T} C^{-1} B}=C-\frac{B B^{T}}{a},
$$

поскольку $r=a-B^{T} C^{-1} B$. Первая часть доказана. 
Теперь докажем вторøе утверждение. Проделаем следующие преобразования определителя. Пусть $B=\left(b_{1}, \ldots, b_{d}\right)^{T}$. Рассмотрим первую строку матрицы $\left(\begin{array}{cc}a & B^{T} \\ B & C\end{array}\right)$, домножим. ее на $b_{i} / a$ и вычтем результат из $(i+1)$-й строки для всех $i=1, \ldots, d$. После этого мы получим матрицу $C-a^{-1} B B^{T}$ на месте $C$, а первый столбец заменится на $(a, 0,0, \ldots, 0)^{T}$. Разлагая полученный определитель по первому столбцу, получаем результат леммы.

Теперь мы готовы закончить доказательство теоремы 2. В силу теоремы 3 и равенства (4) знаменатель правой части соотношения (1) сокрапцается до $\left(\sqrt{2 \pi \mathbf{D}_{x} \mathbf{n}}\right)^{-1}(1+O(\sigma))$. Проследим, как можно сократить числитель. Мы также заменяем его асимптотическим выражением, данным теоремой 3 . Согласно теореме 3 , с учетом соотношения (4), получим

$$
\begin{aligned}
\mu_{x} & \left\{\lambda \in \mathscr{P}: \mathrm{n}(\lambda)=n, \hat{g}_{\lambda}(\mathbf{t})=M\right\} \\
& =\frac{1}{(2 \pi)^{(d+1) / 2} \sqrt{|D|}} \exp \left(-\frac{\left\langle\bar{N}, D^{-1} \bar{N}\right\rangle}{2}\right)(1+O(\sigma)) .
\end{aligned}
$$

Используя лемму 1 , видим, что $\bar{N}=\left(O(\sigma), m_{1}-g\left(t_{1}\right) / \sigma+O(1)\right.$, $\left.m_{2}-g\left(t_{2}\right) / \sigma+O(1), \ldots, m_{d}-g\left(t_{d}\right)+O(1)\right)$. Обозначим

$$
\left(\begin{array}{cc}
\tilde{a} & \widetilde{B}^{T} \\
\widetilde{B} & \widetilde{C}
\end{array}\right)=\left(\begin{array}{cc}
a & B^{T} \\
B & C
\end{array}\right)^{-1}=D^{-1}=\left(\begin{array}{cc}
\mathbf{D}_{x} \mathbf{n} & \operatorname{Cov}[\mathbf{n}, \widehat{g}(\mathbf{t})] \\
\operatorname{Cov}[\mathbf{n}, \widehat{g}(\mathbf{t})] & \mathbf{D}_{x}(\widehat{g}(\mathbf{t}))
\end{array}\right)^{-1}
$$

(здесь $a, \widetilde{a}, B, \widetilde{B}, C, \widetilde{C}$ такие, как описано в формулировке леммы 2). Поэтому скалярное произведение в правой части (8) равняется

$$
\left\langle M-\mathbf{E}_{x} \widehat{g}_{\lambda}(\mathbf{t}), \widetilde{C}\left(M-\mathbf{E}_{x} \widehat{g}_{\lambda}(\mathbf{t})\right)\right\rangle+O(\sigma) .
$$

Таким образом, показатель экспоненты в правой части (8) асимптотически совпадает с показателем экспоненты в плотности нормального распределения в $\mathbf{R}^{2}$ со средним $\sigma^{-1} \widehat{g}(\mathrm{t})$ и матрицей ковариаций $\widetilde{C}^{-1}$. Чтобы найти $\widetilde{C}^{-1}$, мы применяем лемму 2 , которая дает следующее выражение:

$$
\tilde{C}^{-1}=C-\frac{1}{a} B B^{T}=\mathbf{D}_{x} \widehat{g}_{\lambda}(\mathbf{t})-\frac{1}{\mathbf{D}_{x} \mathbf{n}} \operatorname{Cov}\left[\mathbf{n}, \widehat{g}_{\lambda}(\mathrm{t})\right] \operatorname{Cov}\left[\mathbf{n}, \widehat{g}_{\lambda}(\mathbf{t})\right]^{T} .
$$

Теперь остается лишь подставить в (1) асимптотические выражения, найденные в лемме 1 , и использовать разложение определителя $|D|$, полученное в лемме 2. Проделаем эти вычисления:

$$
\begin{aligned}
\mu^{n} & \left\{\lambda \in \mathscr{P}(n): \widehat{g}_{\lambda}(\mathrm{t})=M\right\} \\
= & \frac{\left((2 \pi)^{(d+1) / 2} \sqrt{|D|}\right)^{-1} \exp \left(-\left\langle\bar{N}, D^{-1} \bar{N}\right\rangle / 2\right)(1+O(\sigma))}{\left(\sqrt{2 \pi \mathbf{D}_{x} \mathbf{n}}\right)^{-1}(1+O(\sigma))}
\end{aligned}
$$




$$
\begin{aligned}
= & \frac{1}{(2 \pi)^{d / 2} \sqrt{\left|C-a^{-1} B B^{T}\right|}} \\
& \times \exp \left(-\frac{1}{2}\left\langle M-\frac{1}{\sigma} g(\mathbf{t}),\left(C-\frac{1}{a} B B^{T}\right)^{-1}\left(M-\frac{1}{\sigma} g(\mathbf{t})\right)\right)\right) .
\end{aligned}
$$

Вычисляя $C-a^{-1} B B^{T}$ с использованием леммы 1 , получаем утверждение теоремы 2.

4. Локальная предельная теорема в большом ансамбле разбиений. Докажем теперь теорему 3. Для того чтобы пояснить ее сходство с локальной предельной теоремой, переформулируем ее в других терминах. Будем рассматривать лишь меры $\mu_{x}$ на $\mathscr{P}, x \in(0,1)$.

Пусть $d \in \mathbf{Z}_{+}$. Рассмотрим $d$ неотрицательных вещественных чисел $0 \leqslant t_{1}<t_{2}<\cdots<t_{d}<\infty$. Рассмотрим последовательность случайных $(d+1)$-мерных векторов $\xi_{k}$, зависящих от разбиения $\lambda \in \mathscr{P}$ :

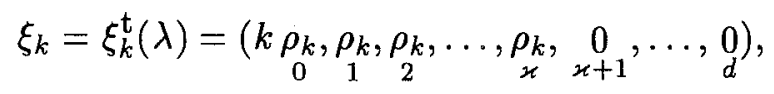

где $\varkappa=\varkappa(k)$ определено соотношением $k \sigma \in\left[t_{\varkappa}, t_{\varkappa+1}\right), \sigma=\sigma(x)=-\ln x$, и $t_{d+1}$ предполагается равным $+\infty$. Таким образом, число компонент вектора $\xi_{k}$, которые не тождественно равны нулю, есть $\varkappa+1$, а $\varkappa$ есть число точек $t_{i}$, меньших, чем $\sigma k$. (Таким образом, $\varkappa$ зависит от $x$ и $k$ и не убывает, когда $k \rightarrow \infty$ при фиксированном $x$.) Про случайные величины $\rho_{k}$ мы предполагаем, что они имеют распределение, индуцированное мерой $\mu_{x}$. Таким образом, при фиксированном наборе чисел $t_{1}, \ldots, t_{d}$ распределение $\xi_{k}$ полностью определяется числом $x$.

Сумма $\xi=\sum_{k=1}^{\infty} \xi_{k}$ имеет следующий смысл: ее первая компонента $\xi^{(0)}$ есть разбиваемое число, а компонента $\xi^{(j)}, j \geqslant 1$, 一 число слагаемых в разбиении $\left(\rho_{k}\right)$, не меньших $t_{j} / \sigma$. В обозначениях предыдушего пункта $\xi(\lambda)=\left(\mathbf{n}(\lambda), \widehat{g}_{\lambda}(\mathbf{t})\right)$.

Рассмотрим матрицу ковариаций $D_{x}=D_{x}(\mathbf{t})=\mathbf{D}_{x} \xi$ случайного вектора $\xi$. Лемма 1 утверждает, что при $x \rightarrow 1$

где

$$
\mathbf{D}_{x}=\left(\begin{array}{ccccc}
\frac{1}{\sigma^{3}} \frac{\pi^{2}}{6} & \frac{1}{\sigma^{2}} h\left(t_{1}\right) & \frac{1}{\sigma^{2}} h\left(t_{2}\right) & \cdots & \frac{1}{\sigma^{2}} h\left(t_{d}\right) \\
\frac{1}{\sigma^{2}} h\left(t_{1}\right) & \frac{1}{\sigma} \frac{1}{1+e^{t_{1}}} & \frac{1}{\sigma} \frac{1}{1+e^{t_{2}}} & \cdots & \frac{1}{\sigma} \frac{1}{1+e^{t_{d}}} \\
\frac{1}{\sigma^{2}} h\left(t_{2}\right) & \frac{1}{\sigma} \frac{1}{1+e^{t_{2}}} & \frac{1}{\sigma} \frac{1}{1+e^{t_{2}}} & \cdots & \frac{1}{\sigma} \frac{1}{1+e^{t_{d}}} \\
\vdots & \vdots & \vdots & \ddots & \vdots \\
\frac{1}{\sigma^{2}} h\left(t_{d}\right) & \frac{1}{\sigma} \frac{1}{1+e^{t_{d}}} & \frac{1}{\sigma} \frac{1}{1+e^{t_{d}}} & \cdots & \frac{1}{\sigma} \frac{1}{1+e^{t_{d}}}
\end{array}\right)(1+O(\sigma))
$$

$$
\sigma=-\ln x \quad \text { и } \quad h(t)=\frac{t}{1+e^{t}}+\ln \left(1+e^{-t}\right) .
$$


Ниже мы докажем локальную предельную теорему для последовательности $\xi_{k}$ при $x \rightarrow 1$. Приведем еше раз точную формулировку в новых терминах.

Теорема 3. Пусть $N=N(x)=\left(n, m_{1}, \ldots, m_{d}\right)$ есть $(d+1)$-мерный вектор с неотричательными челыми компонентами, зависящий от $x$ max, что $n / \ln ^{2} x \in[a, b], m_{i} / \ln x \in[a, b], 0<a<b<\infty$. Обозначим $\bar{N}=N-\mathbf{E}_{x} \xi$. Тогда при любых $а$ и $и$

$$
\begin{aligned}
& \left|\mu_{x}\{\lambda \in \mathscr{P}: \xi(\lambda)=N\}-\frac{1}{(2 \pi)^{(d+1) / 2} \sqrt{\left|D_{x}\right|}} \exp \left(-\frac{\left\langle\bar{N}, D_{x}^{-1} \bar{N}\right\rangle}{2}\right)\right| \\
& \quad=O(|\ln x|),
\end{aligned}
$$

прu $x \rightarrow 1$.

Д о к а з а те ль с т в о. Прежде всего введем необходимые нам обозначения. Характеристическая функция случайной величины $\rho_{k}$ будет обозначаться $\psi_{k}(\beta)=\mathbf{E}_{x} \exp \left\{i \beta \rho_{k}\right\}$, а характеристическая функция центрированной случайной величины $\bar{\rho}_{k}=\rho_{k}-\mathbf{E}_{x} \rho_{k}-$ тем же символом с чертой: $\bar{\psi}_{k}(\beta)$. Нетрудно проверить, что в нашем случае

$$
\psi_{k}(\beta)=\frac{1+x^{k} e^{i k \beta}}{1+x^{k}} \quad \text { и } \quad \bar{\psi}_{k}(\beta)=\frac{1+x^{k} e^{i k \beta}}{1+x^{k}} e^{-i k x^{k} /\left(1+x^{k}\right)} .
$$

Характеристическая функция случайного вектора $\xi_{k}$ будет обозначаться $\varphi_{k}\left(\alpha_{0}, \ldots, \alpha_{d}\right)=\mathbf{E}_{x} \exp \left\{i\left\langle\alpha, \xi_{k}\right\rangle\right\} ;$ центрированного случайного вектора $\bar{\xi}_{k}=\xi_{k}-\mathbf{E}_{x} \xi_{k}-$ тем же символом с чертой: $\bar{\varphi}_{k}\left(\alpha_{0}, \ldots, \alpha_{d}\right)$. Мы пишем $\alpha=\left(\alpha_{0}, \ldots, \alpha_{d}\right)$ для краткости. ІІростое вычисление показывает, что $\varphi_{k}(\alpha)=\psi_{k}\left(\beta_{k}(\alpha)\right)$ и $\bar{\varphi}_{k}(\alpha)=\bar{\psi}_{k}\left(\beta_{k}(\alpha)\right)$, где $\beta_{k}(\alpha)=$ $k \alpha_{0}+\alpha_{1}+\alpha_{2}+\cdots+\alpha_{\varkappa}$, где $\varkappa$, как и прежде, определено соотношением $\sigma k \in\left[t_{\varkappa}, t_{\varkappa+1}\right)$. Теорема умножения для характеристических функций показывает, что $\varphi(\alpha)=\prod_{k=1}^{\infty} \varphi_{k}(\alpha)$ и $\bar{\varphi}(\alpha)=\prod_{k=1}^{\infty} \bar{\varphi}_{k}(\alpha)$.

Пусть $B_{x}$ есть симметричная положительно определенная матрица такая, что $B_{x}^{2}=D_{x}^{-1}$. Заметим, что $\sqrt{\left|D_{x}\right|}=\left|B_{x}^{-1}\right|=\left|B_{x}\right|^{-1}$, где $|\cdot|$ обозначает определитель.

Следуюшие символы будут обозначать подмножества $\mathbf{R}^{d+1}$ :

(1) $A^{b}=\left\{\alpha \in \mathbf{R}^{d+1}:\left|\alpha_{j}\right| \leqslant b, j=0,1, \ldots, d\right\}$,

(2) $A^{b_{0}, b_{1}}=\left\{\alpha \in \mathbf{R}^{d+1}:\left|\alpha_{0}\right| \leqslant b_{0},\left|\alpha_{j}\right| \leqslant b_{1}, j=1, \ldots, d\right\}$,

(3) $A_{a}^{b}=A^{b} \backslash A^{a}$, где подразумевается $a<b$,

(4) $A_{a_{0}, a_{1}}^{b_{0}, b_{1}}=A^{b_{0}, b_{1}} \backslash A^{a_{0}, a_{1}}$, где $a_{0}<b_{0}, \alpha_{1}<b_{1}$;

и следуюшие - их прообразы при отображении $\alpha \mapsto B_{x} \alpha$ :

(1) $C^{b}=B_{x}^{-1} A^{b}=\left\{\alpha \in \mathbf{R}^{d+1}: B_{x} \alpha \in A^{b}\right\}$

(2) $C^{b_{0}, b_{1}}=B_{x}^{-1} A^{b_{0}, b_{1}}=\left\{\alpha \in \mathbf{R}^{d+1}: B_{x} \alpha \in A^{b_{0}, b_{1}}\right\}$,

(3) $C_{a}^{b}=B_{x}^{-1} A_{a}^{b}=\left\{\alpha \in \mathbf{R}^{d+1}: B_{x} \alpha \in A_{a}^{b}\right\}$,

(4) $C_{a_{0}, a_{1}}^{b^{0}, b^{1}}=B_{x}^{-1} A_{a_{0}, a_{1}}^{b_{0}, b_{1}}=\left\{\alpha \in \mathbf{R}^{d+1}: B_{x} \alpha \in A_{a_{0}, a_{1}}^{b_{0}, b_{1}}\right\}$.

Мы также пишем $d \alpha=d \alpha_{0} d \alpha_{1} \cdots d \alpha_{d}$. Отметим, что ниже все интегралы относительно $\alpha$ берутся в $\mathbf{R}^{d+1}$. 
В нашем доказательстве мы будем следовать, в целом, обычной схеме доказательства локальных предельных теорем, которая может быть найдена, в многомерном случае, в [5]. Положим

$$
y=B_{x}\left(N-\mathbf{E}_{x} \xi\right)
$$

Обозначим левую часть (9) символом $R_{x}$, так что в новых обозначениях

$$
\sqrt{\left|D_{x}\right|} R_{x}=\left|\sqrt{\left|D_{x}\right|} \mu_{x}\left\{B_{x}\left(\xi-\mathbf{E}_{x} \xi\right)=y\right\}-\frac{1}{(2 \pi)^{(d+1) / 2}} e^{-\langle y, y\rangle / 2}\right|
$$

По формуле обращения для характеристических функций имеем

$$
\begin{aligned}
& \sqrt{\left|D_{x}\right|} \mu_{x}\{\xi=N\}=\sqrt{\left|D_{x}\right|} \mu_{x}\left\{B_{x}\left(\xi-\mathbf{E}_{x} \xi\right)=y\right\} \\
& =\frac{\sqrt{\left|D_{x}\right|}}{(2 \pi)^{d+1}} \int_{A^{\pi}} \varphi(\alpha) e^{-i\left\langle\alpha, B_{x}^{-1} y+\mathbf{E}_{x} \xi\right\rangle} d \alpha=\frac{\sqrt{\left|D_{x}\right|}}{(2 \pi)^{d+1}} \int_{A^{\pi}} \bar{\varphi}(\alpha) e^{-i\left\langle B_{x}^{-1} \alpha, y\right\rangle} d \alpha \\
& =\frac{1}{(2 \pi)^{d+1}} \int_{C^{\pi}} \bar{\varphi}\left(B_{x} \alpha\right) e^{-i\langle\alpha, y\rangle} d \alpha
\end{aligned}
$$

Используя формулу преобразования Фурье нормального распределения, имеем также

$$
\frac{1}{(2 \pi)^{(d+1) / 2}} e^{-\langle y, y\rangle / 2}=\frac{1}{(2 \pi)^{d+1}} \int_{\mathbf{R}^{d+1}} e^{-\langle\alpha, \alpha\rangle / 2-i(\alpha, y)} d \alpha .
$$

Мы должны оценить модуль разности правых частей (11) и (12) при $x \rightarrow 1$ (или, другими словами, при $\sigma \rightarrow 0$ ).

Рассмотрим два числа, зависящих от $x: \varepsilon^{0}(x)=\sigma^{3 / 2} \ln ^{2} \sigma$ и $\varepsilon^{1}(x)=$ $\sigma^{1 / 2} \ln ^{4} \sigma$ (где $\sigma=-\ln x$ в соответствии с уравнением (4)). Для краткости мы будем писать $\varepsilon_{x}^{0}=\varepsilon^{0}(x)$ и $\varepsilon_{x}^{1}=\varepsilon^{1}(x)$. Заметим, что и $\varepsilon_{x}^{0}$, и $\varepsilon_{x}^{1}$ стремятся к нулю при $x \rightarrow 1$, хотя они имеют различные порядки. Мы предполагаем ниже, что $x$ достаточно близко к 1 , так что $\varepsilon_{x}^{i}<\pi$. В таком случае мы можем написать

$$
\begin{aligned}
& (2 \pi)^{d+1} \sqrt{\left|D_{x}\right|} R_{x}=\left|\int_{C^{\pi}} \bar{\varphi}\left(B_{x} \alpha\right) e^{-i(\alpha, y\rangle} d \alpha-\int_{\mathbf{R}^{d+1}} e^{-\langle\alpha, \alpha\rangle / 2-i\langle\alpha, y\rangle} d \alpha\right| \\
& \leqslant\left|\int_{C^{\varepsilon_{x}^{0}, \varepsilon_{x}^{1}}}\left(\bar{\varphi}\left(B_{x} \alpha\right)-e^{-\langle\alpha, \alpha\rangle / 2}\right) e^{-i\langle\alpha, y\rangle} d \alpha\right|
\end{aligned}
$$

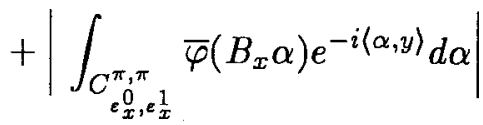

$$
\begin{aligned}
& +\left|\int_{C_{\varepsilon_{x}^{0, \infty}, \frac{1}{x}}^{\infty}} e^{-\langle\alpha, \alpha\rangle / 2-i\langle\alpha, y\rangle} d \alpha\right| \leqslant \int_{C^{\varepsilon_{x}^{0}, \varepsilon_{x}^{1}}}\left|\bar{\varphi}\left(B_{x} \alpha\right)-e^{-\langle\alpha, \alpha) / 2}\right| d \alpha
\end{aligned}
$$

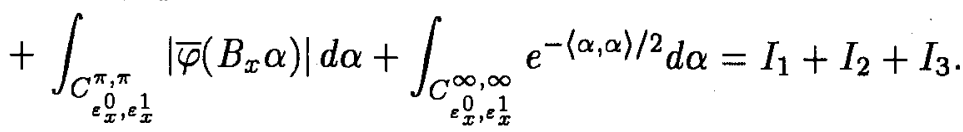


Оценим по отдельности интегралы $I_{1}, I_{2}$ и $I_{3}$. Наиболее просто оценить $I_{3}$ :

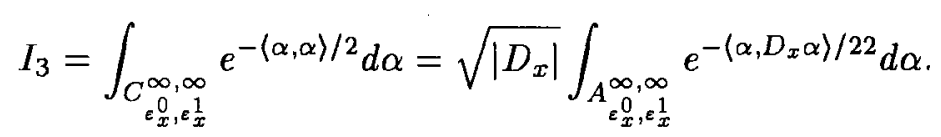

Обозначим

$$
D_{x}=\left(\begin{array}{cc}
\frac{1}{\sigma^{3}} \cdot a & \frac{1}{\sigma^{2}} B^{T} \\
\frac{1}{\sigma^{2}} B & \frac{1}{\sigma} C
\end{array}\right)(1+O(\sigma)) .
$$

(Здесь $a \in \mathbf{R}, B \in \mathbf{R}^{d}$ и $C$ - вещественная матрица $d \times d$, и они не зависят от $\sigma$. .) Также введем обозначения: матрица $\hat{D}_{x}=\left(\begin{array}{cc}a & B^{T} \\ B & C\end{array}\right)$ и матрица $\widehat{B}_{x}-$ симметричная положительно определенная матрица такая, что $\widehat{B}_{x}^{2}=\widehat{D}_{x}^{-1}$. Сделаем замену переменной $\alpha_{0}^{\prime}=\alpha_{0} \sigma^{-3 / 2}$ и $\alpha_{j}^{\prime}=$ $\alpha_{j} \sigma^{-1 / 2}$ при $j=1, \ldots, d$. Как нетрудно проверить, $\left\langle\alpha, D_{x} \alpha\right\rangle=\left\langle\alpha^{\prime}, \widehat{D}_{x} \alpha^{\prime}\right\rangle$ и $\left|D_{x}\right|=\sigma^{-(d+3)}\left|\widehat{D}_{x}\right|$. Поэтому в новых переменных можно записать

$$
\begin{aligned}
I_{3} & =\sqrt{\left|\widehat{D}_{x}\right|} \int_{A_{\ln ^{2} \sigma, \ln ^{4} \sigma}^{\infty, \infty}} e^{-\left\langle\alpha^{\prime}, \widehat{D}_{x} \alpha^{\prime}\right\rangle / 2} d \alpha^{\prime}=\int_{\widehat{C}_{\ln ^{2} \sigma, \ln }^{\infty}{ }^{4} \sigma} e^{-\left\langle\alpha^{\prime}, \alpha^{\prime}\right\rangle / 2} d \alpha^{\prime} \\
& \leqslant \int_{A_{c_{1} \ln ^{2} \sigma}^{\infty}} e^{-\left\langle\alpha^{\prime}, \alpha^{\prime}\right\rangle / 2} d \alpha^{\prime}=\int_{\mathbf{R}^{d+1}} e^{-\left\langle\alpha^{\prime}, \alpha^{\prime}\right\rangle / 2} d \alpha^{\prime}-\int_{A^{c_{1} \ln ^{2} \sigma}} e^{-\left\langle\alpha^{\prime}, \alpha^{\prime}\right\rangle / 2} d \alpha^{\prime} \\
& =(2 \pi)^{(d+1) / 2}-\left(\sqrt{2 \pi}-\frac{2}{c_{1} \ln ^{2} \sigma} e^{-\left(c_{1}^{2} \ln ^{4} \sigma\right) / 2}\left(1+O\left(\frac{1}{\ln ^{4} \sigma}\right)\right)\right)^{d+1} \\
& =(2 \pi)^{d / 2} \frac{2(d+1)}{c_{1} \ln ^{2} \sigma} e^{-\left(c_{1}^{2} \ln ^{4} \sigma\right) / 2}\left(1+O\left(\frac{1}{\ln ^{4} \sigma}\right)\right),
\end{aligned}
$$

где, во-первых, множества $\widehat{C}^{b}, \hat{C}_{a}^{b}, \hat{C}^{b_{0}, b_{1}}$ и $\widehat{C}_{a_{0}, a_{1}}^{b_{0}, b_{1}}$ определяются так же, как. и $C^{b}, C_{a}^{b}, C^{b_{0}, b_{1}}$ и $C_{a_{0}, a_{1}}^{b_{0}, b_{1}}$ соответственно, но в определениях вместо $B_{x}$ стоит $\widehat{B}_{x}$, и, во-вторых, константа $c_{1}>0$ такова, что $A^{c_{1} \ln ^{2} \sigma} \subset \widehat{C}^{\ln ^{2} \sigma, \ln ^{4} \sigma}$. В предпоследней строчке (13) мы использовали асимптотическую формулу

$$
\int_{y}^{\infty} e^{-\beta^{2} / 2} d \beta=\frac{1}{y} e^{-y^{2} / 2}\left(1+O\left(\frac{1}{y^{2}}\right)\right)
$$

см., например, $[17, \S 5.2]$. Заметим, что $\widehat{D}_{x}$ и $\widehat{B}_{x}$ были введены для того, чтобы избежать нахождения асимптотики матрицы $B_{x}$; если бы она была известна, то интеграл $I_{3}$ можно было бы оценить еще проще.

Оценим теперь интеграл $I_{2}$. Прежде всего, преобразуем его следующим образом:

$$
I_{2}=\int_{B_{x}^{-1} A_{\substack{0 \\ \varepsilon_{x}^{0}, \varepsilon_{x}^{1}}}^{\pi, \pi}}\left|\varphi\left(B_{x} \alpha\right)\right| d \alpha=\sqrt{\left|D_{x}\right|} \int_{\substack{A_{\varepsilon_{x}}^{\pi, \pi} \varepsilon_{x}^{1} \\ \varepsilon_{x}^{0}}}|\varphi(\alpha)| d \alpha
$$




$$
\begin{aligned}
& =\sqrt{\left|D_{x}\right|} \int_{\substack{A_{\varepsilon_{x}, \varepsilon_{x}^{1}}^{\pi, \pi} \\
\varepsilon_{x=1}^{0}}}\left|\prod_{k=1}^{\infty} \varphi_{k}(\alpha)\right| d \alpha
\end{aligned}
$$

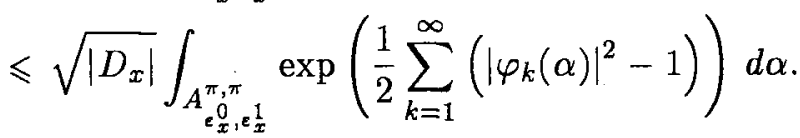

Последняя строчка справедлива в силу неравенства $|z| \leqslant \exp \left\{\frac{1}{2}\left(|z|^{2}-1\right)\right\}$, выполненного при всех $z \in \mathbf{C}$. Вспомним теперь выражение для $\varphi_{k}$ и преобразуем его к виду, удобному для использования в (14):

$$
\begin{aligned}
\left|\varphi_{k}(\alpha)\right|^{2}-1 & =\left|\psi_{k}\left(\beta_{k}(\alpha)\right)\right|^{2}-1=\frac{\left|1+x^{k} e^{i \beta_{k}(\alpha)}\right|^{2}}{\left(1+x^{k}\right)^{2}}-1 \\
& =\frac{\left(1+x^{k} \cos \beta_{k}(\alpha)\right)^{2}+x^{k} \sin ^{2} \beta_{k}(\alpha)-\left(1+x^{k}\right)^{2}}{\left(1+x^{k}\right)^{2}} \\
& =\frac{2 x^{k}\left(\cos \beta_{k}(\alpha)-1\right)}{\left(1+x^{k}\right)^{2}}=\frac{-4 x^{k} \sin ^{2} \frac{\beta_{k}(\alpha)}{2}}{\left(1+x^{k}\right)^{2}}
\end{aligned}
$$

Необходимо показать, что модуль суммы по $k$ таких выражений достаточно велик при всех $\alpha \in A_{\varepsilon_{x}^{0}, \varepsilon_{x}^{1}}^{\pi, \pi}$. Для этого разобъем область $A_{\varepsilon_{x}^{0}, \varepsilon_{x}^{1}}^{\pi, \pi}$ на две части, в зависимости от модуля $\alpha_{0}$, следуюшим образом: $I_{2}=I_{2}^{\prime}+I_{2}^{\prime \prime}$, где $I_{2}^{\prime}$ берется по области $A^{\prime}=\left\{\alpha \in A_{\varepsilon_{x}^{0}, \varepsilon_{x}^{1}}^{\pi, \pi}:\left|\alpha_{0}\right| \geqslant \varepsilon_{x}^{0}\right\}=\left\{\alpha \in \mathbf{R}^{d+1}: \varepsilon_{x}^{0} \leqslant\right.$ $\left.\left|\alpha_{0}\right| \leqslant \pi,\left|\alpha_{j}\right| \leqslant \pi, j=1, \ldots, d\right\}, \mathbf{a} I_{2}^{\prime \prime}-$ по области $A^{\prime \prime}=\{\alpha \epsilon$ $\left.A_{\varepsilon_{x}^{0}, \varepsilon_{x}^{1}}^{\pi, \pi}:\left|\alpha_{0}\right| \leqslant \varepsilon_{x}^{0}\right\}=\left\{\alpha \in \mathbf{R}:\left|\alpha_{0}\right| \leqslant \varepsilon_{x}^{0}, \exists j \in\{1, \ldots, d\}: \varepsilon_{x}^{1} \leqslant\left|\alpha_{j}\right| \leqslant \pi\right\}$. Сперва получим оценку $I_{2}^{\prime}$.

Множество $A^{\prime}$ распадается на две связных компоненты, в которых $\alpha_{0}$ имеет различные знаки. Предположим, что $\alpha_{0}>0 ;$ второй случай рассматривается аналогично. Фиксируем $\alpha \in A^{\prime}, \alpha_{0}>0$, и выясним, при каких $k$ выполняется неравенство $\sin ^{2}\left(\beta_{k}(\alpha) / 2\right) \geqslant \frac{1}{2}$. Для этого необходимо, чтобы

$$
\beta_{k}(\alpha) \in\left[2 \pi p+\frac{\pi}{2}, 2 \pi p+\frac{3 \pi}{2}\right]
$$

при некотором целом числе $p$. Нетрудно проверить, что это так при $k$ между $k^{0}(p)=\left[\left((4 p+1) \pi / 2-\alpha_{1}-\cdots-\alpha_{\varkappa}\right) / \alpha_{0}\right]+1$ и $k^{1}(p)=$ $\left[\left((4 p+3) \pi / 2-\alpha_{1}-\cdots-\alpha_{\varkappa}\right) / \alpha_{0}\right]$, где $[z]$ обозначает целую часть $z$, а $\varkappa=\varkappa(k)$ определено, как и выше, условием $k \sigma \in\left[t_{\varkappa}, t_{\varkappa+1}\right)$. Заметим, что мы молчаливо предполагали, что $\varkappa^{0}\left(k^{0}(p)\right)=\varkappa\left(k^{1}(p)\right)$. Это предположение определенно выполнено, когда $k$ настолько велико, что $\varkappa(k)=d$, или, другими словами, $k \geqslant t_{d} / \sigma$. Это, в свою очередь, верно, если $k^{0}(p) \leqslant k \leqslant k^{1}(p)$ при $p \geqslant t_{d} \alpha_{0} /(2 \pi \sigma)+d / 2 ;$ мы обозначим множество всех таких $k \in \mathbf{N}$ символом $K$. Тем самым, сумма в последней строке 
(14) отрицательна и по модулю н меньше чем

$$
\begin{aligned}
\sum_{k \in K} \frac{4 x^{k}}{\left(1+x^{k}\right)^{2}} \sin ^{2} \frac{\beta_{k}(\alpha)}{2} \geqslant \sum_{k \in K} \frac{x^{k}}{2}=\sum_{p=t_{d} \alpha_{0} /(2 \pi \sigma)+d / 2}^{\infty} \sum_{k=k^{0}(p)}^{k^{1}(p)} \frac{x^{k}}{2} \\
\quad \geqslant \frac{1}{2} \sum_{p=t_{d} \alpha_{0} /(2 \pi \sigma)+d / 2}^{\infty}\left(k^{1}(p)-k^{0}(p)\right) x^{k^{1}(p)} \\
\quad \geqslant C_{1} \frac{\pi}{\alpha_{0}} \frac{x^{k^{1}\left(t_{d} \alpha_{0} /(2 \pi \sigma)+d / 2\right)}}{1-x^{2 \pi / \alpha_{0}}} \\
\quad \geqslant C_{1} \frac{\pi}{\alpha_{0}} \frac{\exp \left(-\sigma\left(4\left(t_{d} \alpha_{0} /(2 \pi \sigma)+d / 2+3\right) \pi / 2+d \pi\right)\right)}{1-\exp \left(2 C_{1} \pi \sigma / \alpha_{0}\right)} \\
\quad=C_{1} \frac{\pi}{\alpha_{0}} \frac{\exp \left(-t_{d} \alpha_{0}-(2 d+3 / 2) \pi \sigma\right)}{1-\left(1-2 C_{1} \pi \sigma / \alpha_{0}+O\left(\sigma^{2} / \alpha_{0}^{2}\right)\right)} \geqslant \frac{C_{2}}{\sigma} .
\end{aligned}
$$

Здесь мы используем проверенное выше утверждение, что $\sin ^{2} \beta_{k}(\alpha) / 2 \geqslant$ $\frac{1}{2}$ при $k \in K$, оценки $k^{1}(p)-k^{0}(p) \geqslant C_{1} \pi / \alpha_{0}, k^{1}(p+1)-k^{1}(p) \geqslant 2 C_{1} \pi / \alpha_{0}$ при некоторой положительной константе $C_{1}$, неравенство $k^{1}(p) \leqslant$ $\left(4\left(t_{d} \alpha_{0} /(2 \pi \sigma)+d / 2+3\right) \pi / 2+d \pi\right)$ и утверждение, что при $\sigma \rightarrow 0 O\left(\sigma^{2} / \alpha_{0}\right)$ стремится к нулю для всех $\alpha \in A^{\prime}$. Отсюда, используя (14), видим, что

$$
I_{2}^{\prime}=O\left(e^{-1 / \sigma}\right) \text {. }
$$

Слагаемое $I_{2}^{\prime \prime}$ отсутствует, если $d=0$, но для случая $d>0$ необходимо также получить оценку для $I_{2}^{\prime \prime}$. Пусть $\alpha \in A^{\prime \prime}$, тогда найдется $j \in\{1, \ldots, d\}$ такое, что $\varepsilon_{x}^{1} \leqslant\left|\alpha_{j}\right| \leqslant \pi$. Пусть $\varkappa_{0} \geqslant 1$ есть минимальное число такое, что $\left|\alpha_{\varkappa_{0}}\right| \geqslant \varepsilon_{x}^{1} / 2^{d-\varkappa_{0}}$. Тогда $\alpha_{1}<\varepsilon_{x}^{1} / 2^{d-1}, \ldots, \alpha_{\varkappa_{0}-1}<$ $\varepsilon_{x}^{1} / 2^{d+1-\varkappa_{0}} ; \pi \geqslant \alpha_{\varkappa_{0}} \geqslant \varepsilon_{x}^{1} / 2^{d-\varkappa_{0}}$, и, суммируя эти неравенства, получаем

$$
\pi+\frac{\varepsilon_{x}^{1}}{2^{d-\varkappa_{0}}} \geqslant\left|\alpha_{1}+\cdots+\alpha_{\varkappa_{0}}\right| \geqslant \frac{\varepsilon_{x}^{1}}{2^{d-1}} .
$$

Обозначим $k_{0}$ минимальное число такое, что $\varkappa\left(k_{0}\right)=\varkappa_{0}$, или, иначе говоря, $k_{0}=\left[t_{\varkappa_{0}} / \sigma\right]$. Теперь нам следовало бы рассмотреть два случая: $\varkappa_{0}<d$ и $\varkappa_{0}=d$, но, чтобы избежать этого, введем также $t_{d+1}=2 t_{d}$, чтобы сделать опенки одинаковыми в обоих случаях. Рассмотрим $k_{1}=\left[t_{\varkappa_{0}+1} / \sigma\right]$. Используя уравнение (15) и вычисления, аналогичные (14) (с измененной областью интегрирования), получаем

$$
\begin{aligned}
I_{2}^{\prime \prime} & \leqslant \int_{A^{\prime \prime}} \exp \left(\frac{1}{2} \sum_{k=1}^{\infty}\left(\left|\varphi_{k}(\alpha)\right|^{2}-1\right)\right) d \alpha \\
& \leqslant \int_{A^{\prime \prime}} \exp \left(-\sum_{k=k_{1}}^{k_{2}} \frac{2 x^{k}}{\left(1+x^{k}\right)^{2}} \sin ^{2} \frac{\beta_{k}(\alpha)}{2}\right) d \alpha .
\end{aligned}
$$

Оценим сумму в последней строке неравенства (18). Принимая во внимание (17), неравенства $\alpha_{0} \leqslant \varepsilon_{x}^{0}, k_{0} \leqslant k \leqslant k_{1}$, и замечая, что $\varkappa_{(k)=\varkappa_{0}}$ 
при таких $k$, получаем следующие ограничения для $\beta_{k}(\alpha)$ :

$$
2^{-d} \sigma^{1 / 2} \ln ^{4} \sigma \leqslant \beta_{k}(\alpha) \leqslant \frac{3 \pi}{2} .
$$

Эти неравенства выполнены при $\sigma$, достаточно близких к нулю, таких, что $\ln ^{2} \sigma \geqslant 2^{d} t_{\varkappa_{0}+1}$. (Заметим, что существенным здесь является ограничение снизу.) При $k \leqslant k_{1}$ мы имеем оценку $x^{k}=e^{-k \sigma} \geqslant \exp \left(-t_{\varkappa_{0}+1}\right)$. Используя ее, неравенство (19), а также оценку

$$
\frac{2 x^{k}}{\left(1+x^{k}\right)^{2}} \sin \frac{\beta_{k}(\alpha)}{2} \geqslant \frac{x^{k}}{200} \beta_{k}(\alpha)^{2}
$$

получаем, что сумма в последней строке (18) не меньше, чем $\left(\exp \left(-t_{\varkappa_{0}+1}\right) / 200\right) \cdot 2^{-2 d} \sigma \ln ^{8} \sigma \cdot\left(t_{\varkappa_{0}+1}-t_{\varkappa_{0}}\right) / \sigma$. Таким образом, комбинируя эту ощенку и (18), получаем

$$
I_{2}^{\prime \prime} \leqslant \int_{A^{\prime \prime}} \exp \left(-C_{3} \ln ^{8} \sigma\right) d \alpha \leqslant C_{4} \sigma^{3 / 2} \ln ^{2} \sigma \exp \left(-\ln ^{8} \sigma\right) .
$$

Теперь осталось оценить интеграл $I_{1}$. ІІрежде всего, преобразуем его:

$$
\begin{aligned}
I_{1} & =\int_{B_{x}^{-1} A^{\varepsilon_{x}^{0}, \varepsilon_{x}^{1}}} \mid \bar{\varphi}\left(B_{x} \alpha\right)-e^{-\langle\alpha, \alpha\rangle / 2 \mid} d \alpha \\
& =\sqrt{\left|D_{x}\right|} \int_{A^{\varepsilon_{x}^{0}, \varepsilon_{x}^{1}}}\left|\bar{\varphi}(\alpha)-e^{-\left\langle\alpha, D_{x} \alpha\right\rangle / 2}\right| d \alpha \\
& \leqslant \sqrt{\left|D_{x}\right|} \int_{A^{e_{x}^{0}, \varepsilon_{x}^{1}}}\left|\bar{\varphi}(\alpha) e^{\left\langle\alpha, D_{x} \alpha\right\rangle / 2}-1\right| d \alpha \\
& =\sqrt{\left|D_{x}\right|} \int_{A^{e_{x}^{0}, \varepsilon_{x}^{1}}}\left|\exp \left(\ln \bar{\varphi}(\alpha)+\frac{1}{2}\left\langle\alpha, D_{x} \alpha\right\rangle\right)-1\right| d \alpha \\
& \leqslant \sqrt{\left|D_{x}\right|} \int_{A^{\varepsilon_{x}^{0}, \varepsilon_{x}^{1}}}\left|\ln \bar{\varphi}(\alpha)+\frac{1}{2}\left\langle\alpha, D_{x} \alpha\right\rangle\right| \\
\times & \exp \left|\ln \bar{\varphi}(\alpha)+\frac{1}{2}\left\langle\alpha, D_{x} \alpha\right\rangle\right| d \alpha .
\end{aligned}
$$

Последнее неравенство выполнено, поскольку $\left|e^{z}-1\right| \leqslant|z| e^{|z|}$ при всех комплексных $z$. Используя разложения

$$
\ln \bar{\varphi}(\alpha)=\sum_{k=1}^{\infty} \ln \bar{\varphi}_{k}(\alpha) \quad \text { и } \quad\left\langle\alpha, D_{x} \alpha\right\rangle=\sum_{k=1}^{\infty} \mathbf{E}_{x}\left\langle\alpha, \bar{\xi}_{k}\right\rangle^{2}
$$

получаем следующее неравенство:

$$
\left|\ln \bar{\varphi}(\alpha)+\frac{1}{2}\left\langle\alpha, D_{x} \alpha\right\rangle\right| \leqslant \sum_{k=1}^{\infty}\left|\ln \bar{\varphi}_{k}(\alpha)+\frac{1}{2} \mathbf{E}_{x}\left\langle\alpha, \bar{\xi}_{k}\right\rangle^{2}\right| .
$$

Обозначим $k$-е слагаемое в правой части $(22)$ символом $T_{k}$. Мы используем формулу $\bar{\varphi}_{k}(\alpha)=\bar{\psi}_{k}\left(\beta_{k}(\alpha)\right)$ и сдедующее разложение $\bar{\psi}_{k}$ : $\bar{\psi}_{k}(\beta)=\frac{1+x^{k} e^{i \beta}}{1+x^{k}} \exp \left(-\frac{i \beta x^{k}}{1+x^{k}}\right)=1-\frac{x^{k}}{\left(1+x^{k}\right)^{2}} \frac{\beta^{2}}{2}+\frac{x^{k}\left(1-x^{k}\right)}{\left(1+x^{k}\right)^{3}} O\left(\beta^{3}\right)$, 
где $O(\cdot)$ может быть выбрано не зависяшим от $x$ и $k$. Мы предполагаем это для всех $O(\cdot)$, встречаюшихся ниже. Отсюда видим, что

$$
\ln \bar{\varphi}_{k}(\alpha)=-\frac{x^{k}}{\left(1+x^{k}\right)^{2}} \frac{\beta_{k}(\alpha)^{2}}{2}+\frac{x^{k}\left(1-x^{k}\right)}{\left(1+x^{k}\right)^{3}} O\left(\beta_{k}(\alpha)^{3}\right) .
$$

Далее, $\mathbf{E}_{x}\left\langle\alpha, \bar{\xi}_{k}\right\rangle^{2}=\beta_{k}(\alpha)^{2} \mathbf{E}_{x}\left(\bar{\rho}_{k}^{2}\right)=x^{k}\left(1+x^{k}\right)^{-2} \beta_{k}(\alpha)^{2}$, и, значит,

$$
T_{k}=\frac{x^{k}\left(1-x^{k}\right)}{\left(1+x^{k}\right)^{3}} O\left(\beta_{k}(\alpha)^{3}\right) \text {. }
$$

Поэтому

$$
\begin{aligned}
T_{k} & \leqslant C_{5} x^{k} \beta_{k}(\alpha)^{3} \leqslant C_{5} x^{k}\left(k^{3}\left(\varepsilon_{x}^{0}\right)^{3}+3 d k^{2}\left(\varepsilon_{x}^{0}\right)^{2} \varepsilon_{x}^{1}+3 d^{2} k \varepsilon_{x}^{0}\left(\varepsilon_{x}^{1}\right)^{2}+d^{3}\left(\varepsilon_{x}^{1}\right)^{3}\right) \\
& \leqslant C_{5} e^{-\sigma k}\left((\sigma k)^{3}+3 d(\sigma k)^{2}+3 d^{2} \sigma k+d^{3}\right) \sigma^{3 / 2} \ln ^{12} \sigma,
\end{aligned}
$$

где константа $C_{5}$ не зависит ни от $x$, ни от $k$. Суммируя последние неравенства, получаем

$$
\begin{aligned}
\sum_{k=1}^{\infty} T_{k} & \leqslant \sum_{k=1}^{\infty} C_{5} e^{-\sigma k}\left((\sigma k)^{3}+3 d(\sigma k)^{2}+3 d^{2} \sigma k+d^{3}\right) \sigma^{3 / 2} \ln ^{12} \sigma \\
& \leqslant C_{6} \sigma^{1 / 2} \ln ^{12} \sigma \int_{0}^{\infty} e^{-z}\left(z^{3}+3 d z^{2}+3 d^{2} z+d^{3}\right) d z \\
& =C_{7}(d) \sigma^{1 / 2} \ln ^{12} \sigma .
\end{aligned}
$$

Отсюда мы заключаем, что выражение $\left|\ln \bar{\varphi}(\alpha)+\frac{1}{2}\left\langle\alpha, D_{x} \alpha\right\rangle\right|$, стояшее в показателе экспоненты в (21), ограничено при $\sigma \rightarrow 0$ и $\alpha \in A^{\varepsilon_{x}^{0}, \varepsilon_{x}^{1}}$. Используя эту оценку, а также (21), (22) и (23), получаем неравенства

$$
\begin{aligned}
I_{1} \leqslant & C_{8} \sqrt{\left|D_{x}\right|} \sum_{k=1}^{\infty} \int_{A^{\varepsilon_{x}^{0}, \varepsilon_{x}^{1}}} T_{k} d \alpha \leqslant C_{9} \sqrt{\left|D_{x}\right|} \sum_{k=1}^{\infty} \frac{x^{k}\left(1-x^{k}\right)}{\left(1+x^{k}\right)^{3}} \int\left|\beta_{k}(\alpha)\right|^{3} d \alpha \\
\leqslant & C_{10} \sqrt{\left|D_{x}\right|} \sum_{k=1}^{\infty} x^{k}\left(1-x^{k}\right) \\
& \times \int_{0}^{\varepsilon_{x}^{0}} d \alpha_{0} \int_{\left[0, \varepsilon_{x}^{1}\right]^{d}} \ldots \int\left(k \alpha_{0}+\alpha_{1}+\cdots+\alpha_{d}\right)^{3} d \alpha_{1} \cdots d \alpha_{d} \\
= & \sqrt{\left|D_{x}\right|} \sum_{k=1}^{\infty} x^{k}\left(1-x^{k}\right) \int_{0}^{\varepsilon_{x}^{0}}\left(C_{10}\left(k \alpha_{0}\right)^{3}\left(\varepsilon_{x}^{1}\right)^{d}+C_{11}\left(k \alpha_{0}\right)^{2}\left(\varepsilon_{x}^{1}\right)^{d+1}\right. \\
& \left.+C_{12}\left(k \alpha_{0}\right)\left(\varepsilon_{x}^{1}\right)^{d+2}+C_{13}\left(\varepsilon_{x}^{1}\right)^{d+3}\right) d \alpha_{0} \\
\leqslant & C_{14} \sqrt{\left|D_{x}\right|} \sigma^{3+d / 2} \ln ^{14+4 d} \sigma=O(\sigma) .
\end{aligned}
$$

Комбинируя неравенства (13), (16), (20) и (24), мы получаем результат теоремы. 


\section{СПИСОК ЛИТЕРАТУРРЫ}

1. Эндрюс Дж. Теория разбиений. М.: Наука, 1982, 255 с.

2. Arratia $R$., Tavaré $S$. Independent process approximation for random combinatorial structures. - Adv. Math., 1994, v. 4, № 1, p. 90-154.

3. Barany $I$. The limit shape of convex lattice polygons. - Discrete Comput. Geom., 1995 , v. 13, p. 279-295.

4. Блиновский В. М. Принцип больших уклонений для границы случайной диаграммы Юнга. - Проблемы передачи информации (в печати).

5. Bhattacharya R. N., Rao R. R. Normal Approximation and Asymptotic Expansions. New York: Wiley, 1976, 274 p.

6. Dembo A., Vershik A., Zeitouni O. Large deviations for integer partitions. - Adv. Appl. Math. (to appear).

7. Невская К.И. (ред.) Неопубликованные материалы Л. Эйлера по теории чисел. СПб.: Наука, 1997.

8. Freiman G. On extremal additive problems of Paul Erdös. - Ars Combin., 1988, v. 26 , p. $93-114$.

9. Freiman G. Asymptotical Formula for a Partition Function $q(n)$. Technical Report 3-98. Tel Aviv: Tel Aviv University, 1998.

10. Freiman G., Pitman J. Partitions into distinct large parts. - J. Austral. Math. Soc., Ser. A, 1994, v. 57, № 3, p. 286-416.

11. Fristedt $B$. The structure of random partitions of large integers. - Trans. Amer. Math. Soc., 1993, v. 337, אo 2, p. 703-735.

12. Хуане К. Статистическая механика. М.: Мир, 1966.

13. Хиячия А.Я. Математические основания статистической механики. М.: Гостехиздат, 1943.

14. Хинчин A.Я. Математические основания квантовой статистики. М.: Гостехиздат, 1951, $256 \mathrm{c}$.

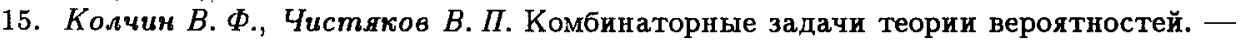
Итоги науки и техники, сер. теор. вер., матем. статист., теор. киберн., 1974, т. 11, c. $5-45$.

16. Ландау Л.Д., Лифиич Е. М. Статистическая физика (классическая и квантовая). М.-JI.: Гостехиздат, 1951.

17. Прохоров Ю. В., Розанов Ю. А. Теория вероятностей. (Основные понятия. Предельные теоремы. Случайные процессы.) М.: Наука, 1987.

18. Сияай Я.Г. Вероятностный подход к анализу статистики выпуклых ломаных. - Функц. анализ и его прил., 1994, т. 28, № 2, с. 41-48.

19. Сачков $B$. $H$. Вероятностные методы в комбинаторном анализе. М.: Наука, 1978, $287 \mathrm{c}$.

20. Вериик $A . M$. Статистическая механика комбинаторных разбиений. - Функц. анализ и его прил., 1996, т. 30, № 2, с. 19-39.

21. Вериик A.M. Предельное распределение энергии квантового идеального газа. с точки зрения теории разбиений натуральных чисел. - Успехи матем. наук, 1997, т. 52, № 2, с. 139-146.

22. Vershik A.M. Asymptotic combinatorics and algebraic analysis. - Proceedings of International Congress of Mathematicians (Zürich, 1994). V. II. Ed. by S. D. Chatterji. Basel: Birkhäuser, 1995, p. 1384-1394.

23. Якубович $О$. В. Асимптотика случайных разбиений множеств. - Записки научн. семин. ПОМИ, 1995, т. 223 , с. 227-250. 